\title{
Aberrant DNA methylation suppresses expression of estrogen receptor 1 (ESR1) in ovarian endometrioma
}

Ryo Maekawa* (D), Yumiko Mihara, Shun Sato, Maki Okada, Isao Tamura, Masahiro Shinagawa, Yuichiro Shirafuta, Haruka Takagi, Toshiaki Taketani, Hiroshi Tamura and Norihiro Sugino

\begin{abstract}
Background: In ovarian endometriomas (OE), the expression statuses of various steroid hormone receptors are altered compared with their expression statuses in eutopic endometrium (EE). For example, in OE, the expressions of estrogen receptor 1 (ESR1), which encodes ERa, and progesterone receptor (PGR) are downregulated, while the expression of ESR2, which encodes ERß, is upregulated. The causes of these changes are unclear. DNA methylation of a specific region of a gene can result in tissue-specific gene expression. Such regions are called tissue-dependent and differentially methylated regions (T-DMRs). We previously reported that the tissue-specific expression of ESR1 is regulated by DNA methylation of a T-DMR in normal tissues. In the present study, we examined whether aberrant DNA methylation of the T-DMR is associated with the altered expressions of ESR1, ESR2 and PGR in OE.
\end{abstract}

Results: Gene expression levels of ESR1, ESR2 and PGR were measured by quantitative RT-PCR. The expression levels of ESR1 and PGR were significantly lower and the expression level of ESR2 was significantly higher in OE than in EE. DNA methylation statuses were examined with an Infinium HumanMethylation450K BeadChip and sodium bisulfite sequencing. DNA methylation at the T-DMRs of ESR1 were significantly higher in OE than in EE, but no significant differences were observed in the DNA methylation statuses of ESR2 and PGR.

Conclusions: Aberrant DNA methylation of the T-DMR was associated with the impaired expression of ESR1, but not the altered expressions of ESR2 and PGR, in OE.

Keywords: Endometriosis, Ovarian endometrioma, Eutopic endometrium, Steroid receptor, Estrogen receptor 1, Estrogen receptor 2, Progesterone receptor, DNA methylation

\section{Background}

Endometriosis is a common gynecological disease affecting approximately $10 \%$ of reproductive age females [1], and is characterized by the ectopic localization of endometrial-like tissue in the pelvic cavity [1]. The disease induces chronic inflammation in the pelvic cavity, leading to symptoms such as chronic pelvic pain and infertility that subsequently affect the patient's health $[1,2]$.

Endometriosis develops mostly in women of reproductive age and regresses after menopause, suggesting that the growth is estrogen-dependent. Estrogen hormone action is mediated by the estrogen receptor in many

\footnotetext{
* Correspondence: rmaekawa@yamaguchi-u.ac.jp

Department of Obstetrics and Gynecology, Yamaguchi University Graduate School of Medicine, Minamikogushi 1-1-1, Ube 755-8505, Japan
}

physiological and pathological processes. The estrogen receptor has two subtypes, estrogen receptors $\alpha$ and $\beta$ (ER $\alpha$ and ER $\beta$ ) encoded by estrogen receptor 1 (ESR1) and 2 (ESR2), respectively [3, 4]. ESR1 is the primary mediator of the estrogenic action, and its expression of ESR1 is higher than that of ESR2 in endometrium [5]. In endometriotic tissue, the expression of ESR1 is strongly suppressed, while ESR2 is upregulated [6]. However, the exact mechanisms of the downregulation of ESR1 and upregulation of ESR2 in endometriotic tissue remain unclear [7, 8].

DNA methylation is a well-known epigenetic mark. DNA methylation, which occurs at CpG sites, interrupts the recognition and binding of transcription factors [9], recruits methyl CpG-binding proteins that interact with transcription repressors [9], and induces chromatin condensation

(c) The Author(s). 2019 Open Access This article is distributed under the terms of the Creative Commons Attribution 4.0 International License (http://creativecommons.org/licenses/by/4.0/), which permits unrestricted use, distribution, and 
A

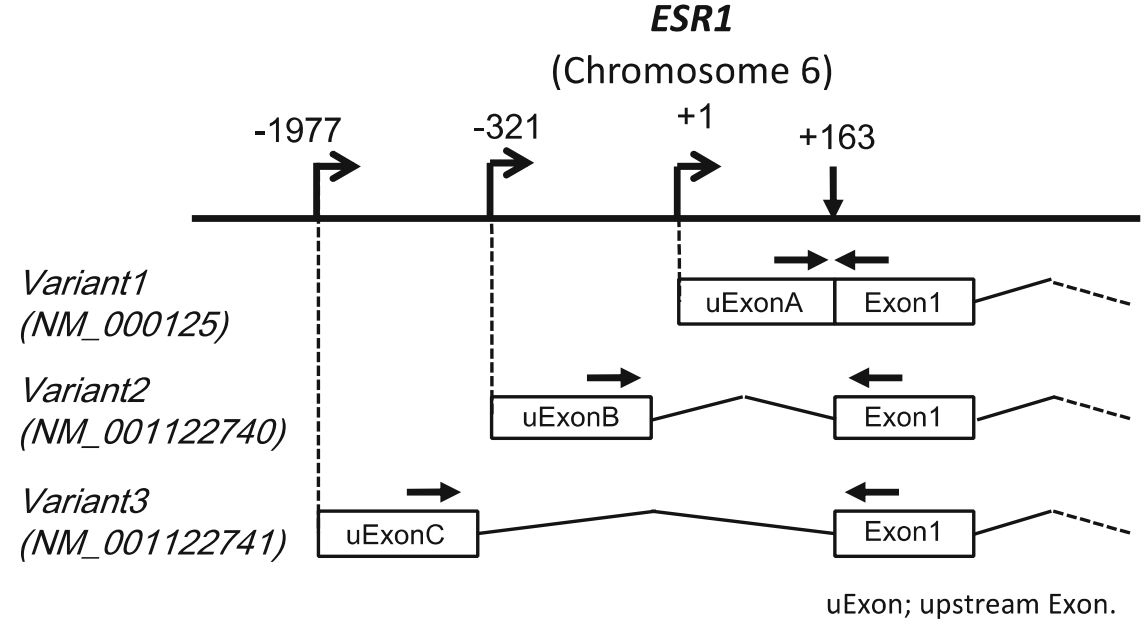

B

ESR2

(Chromosome 14)

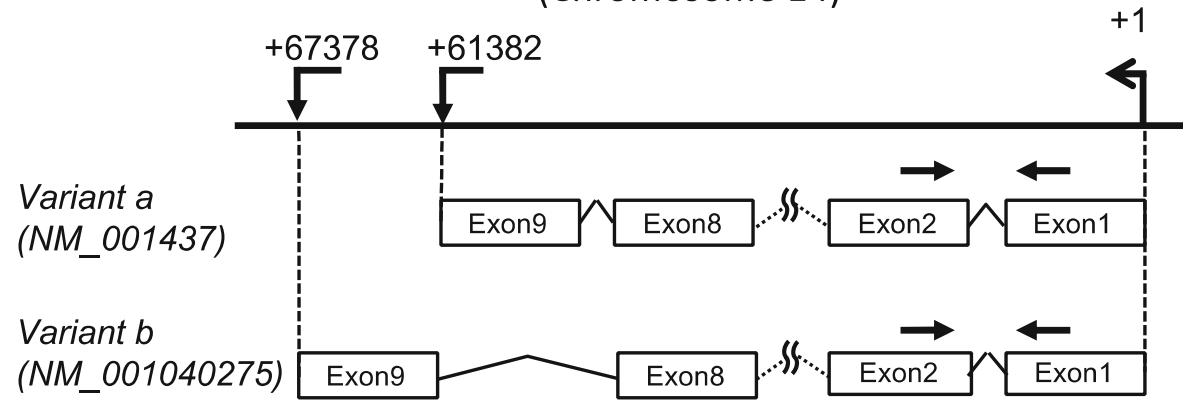

C

PGRs

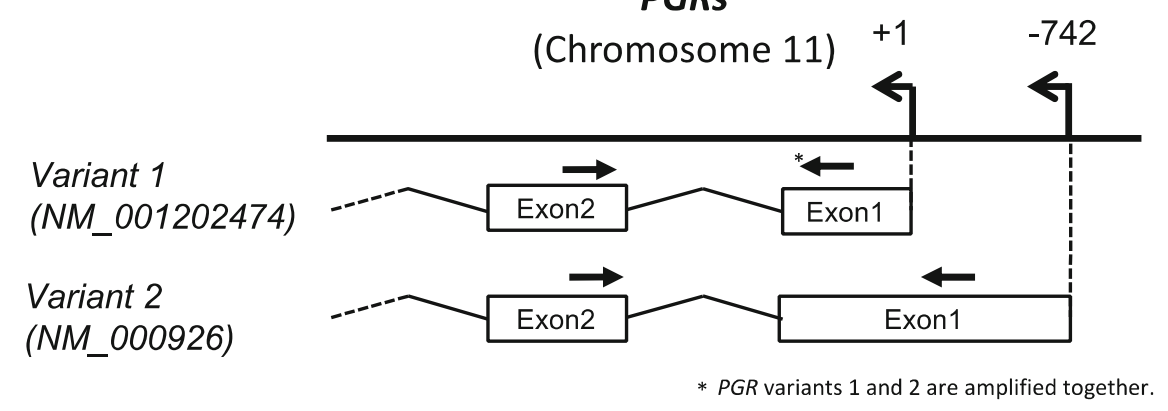

$\longrightarrow$ Spliced region $\quad \longrightarrow \quad \begin{aligned} & \text { Primer pair } \\ & \text { for qRT-PCR }\end{aligned}$

Fig. 1 Genomic organization of ESR1, ESR2 and PGR. a Upstream exons and corresponding transcription start sites (TSSS) of ESR1. The upstream exons are shown by boxes and the corresponding TSSs are indicated by arrows. The numbers show the positions of the 5' start sites of the upstream exons with respect to the start site of upstream Exon A. The different start sites correspond to the mature ESR1 mRNAs, called variant 1 , 2 and 3. All 5' upstream exons are spliced at the common acceptor splice site (+ 163 bp). b Exons, transcription start sites and transcription end sites of ESR2-variant $\mathrm{a}$ and $\mathrm{b}$. The numbers of Exon 9 indicate the distances from the transcription start sites of each variant. $\mathbf{c}$ Exons, transcription start sites of $P G R$-variant 1 and 2. The numbers associated with Exon 1 of variants 1 and 2 indicate the $5^{\prime}$ start sites with respect to the distance from the start site of variant $1(+1)$. The locations of the primer pairs used in GRT-PCR are indicated by the arrows 
via histone modification changes [9]. Maintaining a specific DNA methylation profile in a cell is necessary for cellular integrity, and alterations in DNA methylation may have serious health consequences [9]. We and other groups have previously demonstrated that aberrant epigenetic regulation is associated with the pathogenesis and development of endometriosis [10-13] . In fact, local estrogen production is upregulated in endometriotic tissue by altering the activities of enzymes involved in synthesizing or degrading estradiol $[10,14,15]$. We showed that the altered activities of these genes were caused by aberrant DNA methylation $[10,16]$.

The tissue- and cell-specific expression of a gene can be determined by DNA methylation of a specific region of the gene called the tissue-dependent and differentially methylated region (T-DMR) [17]. Recent genome-wide analyses have identified many T-DMRs in mammalian genomes [1719]. T-DMRs tend to be located at an upstream region of gene promoter or intron rather than in the gene promoter region [18-21]. We previously reported that two T-DMRs are present in the region from $-0.5 \mathrm{~kb}$ to $-1 \mathrm{~kb}$ with respect to the transcription start site of ESR1, and DNA methylation of the two T-DMRs, but not the promoter region, regulate tissue-specific expression of ESR1 in normal tissues [20]. In the tissues with DNA hypermethylation in the two T-DMRs, the expression of ESR1 is suppressed [20]. This led us to hypothesize that the downregulation of ESR1 in endometriosis is caused by aberrant DNA methylation of these T-DMRs. On the other hand, DNA methylation of the promoter region (not the T-DMRs) was reported to regulate the expression of ESR2 [22]. However, because the T-DMRs have a role in ESR1 expression, there is a possibility that regions other than the promoter region are involved in the regulation of ESR2 expression.

Resistance to progesterone may contribute to the pathophysiology of endometriosis [23], and may be caused by aberrant expression of progesterone receptor $(P G R)$ [24]. In cancer tissues, PGR expression may be regulated by DNA methylation of the proximal promoter region of $P G R[25,26]$. However, it remains unclear whether aberrant DNA methylation is also involved in the suppressed expression of $P G R$ in endometriosis.

In the present study, we first compared the expressions of ESR1, ESR2 and PGR in eutopic endometrium (EE) and ovarian endometrioma (OE). Next, we examined the DNA methylation statuses of ESR1, ESR2 and $P G R$ to investigate whether aberrant DNA methylation is associated with their aberrant expressions in $\mathrm{OE}$.

\section{Results}

mRNA expressions of ESR1, ESR2 and PGR

ESR1 has two types of exons: four non-coding upstream-exons (uExons), the most common of which are uExons A, B and C [27], in addition to its coding exons (Exons 1, 2, 3...). The ESR1 mRNA has only one
uExon spliced to the coding exons, so that the transcription start site of ESR1 depends on which uExon is used (Fig. 1a) [27]. The mRNA variants corresponding to uExons $\mathrm{A}, \mathrm{B}$ and $\mathrm{C}$ are called variants 1,2 and 3 , respectively. Because the translation start site (ATG) is located on Exon 1, the uExon does not affect the protein product of ESR1. The mRNA expression statuses of variants 1, 2 and 3 were analyzed in EE and OE by qRT-PCR. Their transcript levels were significantly higher (23.3-, 12.6- and 4.4-folds, respectively) in EE than in OE (Fig. 2a).

ESR2 has two isoforms encoded by ESR2 variants a and $b$ (Fig. 1b). The expression levels of the two variants were examined together using a primer pair that detects both variants. The expression of ESR2 was 134-fold higher in $\mathrm{OE}$ than in EE (Fig. 2b).

$P G R$ has two isoforms, $P R-A$ and $P R-B$, encoded by $P G R$ variants 1 and 2 , respectively (Fig. 1c). Since the primer pair designed for $P G R$ variant 1 also detects $P G R$ variant 2 , the amplified product reflects the combined levels of $P G R$ variants 1 and 2 . The combined expression level of $P G R$ variants 1 and 2 was 12 -fold lower in $\mathrm{OE}$ than in EE (Fig. 2c). The expression of $P G R$ variant 2 was 8-fold lower in OE than in EE (Fig. 2c).

\section{DNA methylation statuses detected by $450 \mathrm{~K}$ BeadChip} ESR1 has two T-DMRs (T-DMR1 and T-DMR2), upstream of the AB-promoter and upstream of the C-promoter, respectively (Fig. 3a) [20]. We first examined the DNA methylation status of the region from $2953 \mathrm{bp}$ to $+229 \mathrm{bp}$ of ESR 1 with the $450 \mathrm{~K}$ BeadChip. DNA methylation was low in the AB-promoter in both EE and OE (Fig. 3a). The DNA methylation statuses of these tissues were not significantly different. On the other hand, the DNA methylation status of T-DMR1 was significantly lower in EE than in OE ( $p<0.05$, Fig. 3a). Similarly, the DNA methylation status in the C-promoter was low in $\mathrm{EE}$ and $\mathrm{OE}$, while the DNA methylation status in T-DMR2 was low in EE and high in OE (Fig. 3a). The DNA methylation status of T-DMR2 was significantly different between EE and $\mathrm{OE}(p<0.05$, Fig. 3a). The DNA methylation status of ESR2 was low in EE and OE and the levels were not significantly different (Fig. 3b). The DNA methylation statuses of PGR-variants 1 and 2 were low and not significantly different in EE and OE (Fig. 3c).

\section{DNA methylation statuses detected by sodium bisulfite sequencing}

In ESR1, DNA methylation levels of the AB- and C-promoters were low in both EE and OE (Fig. 4a). On the other hand, the DNA methylation levels of T-DMR1 and T-DMR2 were low in EE and high in OE (Fig. 4a). In ESR2, DNA methylation was low in both EE and OE (Fig. 4b). 
A
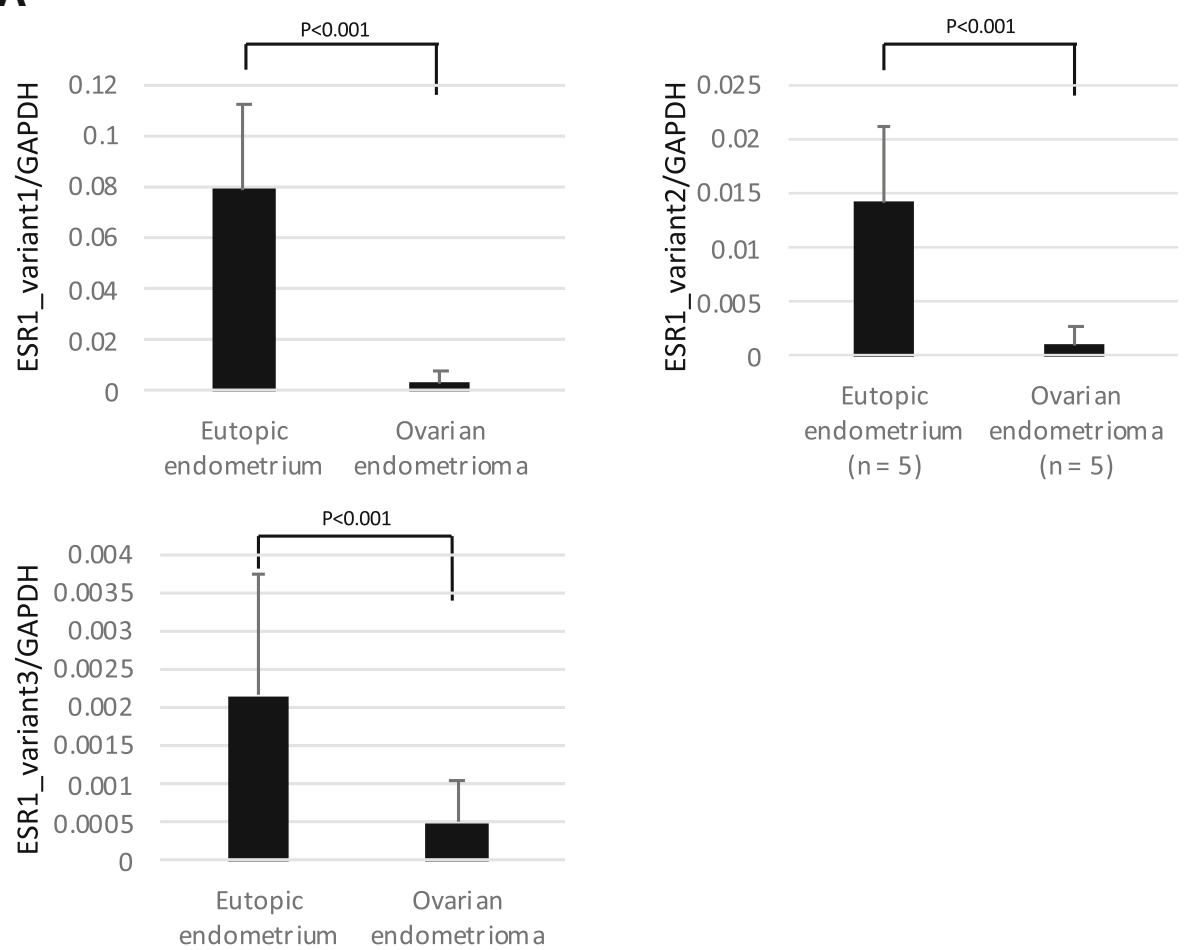

B

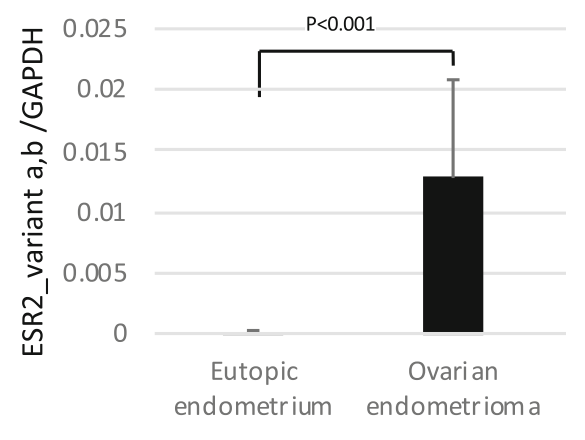

C
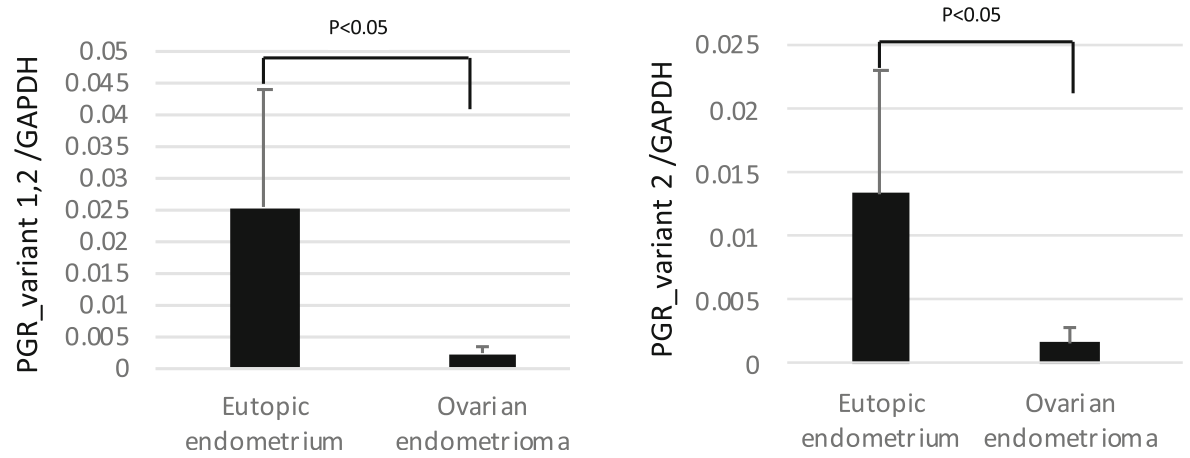

Fig. 2 mRNA expression statuses of ESR1, ESR2 and PGR variants in EE and OE. ESR1 (variant 1, 2, and 3) (a) ESR2 (variant a and b) (b) and PGR (variant 1 and 2) (c) were analyzed by qRT-PCR. GAPDH was used as an internal control. The amount of mRNA of each variant was normalized to that of the internal control (GADPH). Data were expressed as a ratio of mRNA of each variant to GADPH. Each bar represents the mean +/SEM.* $p<0.05$ 


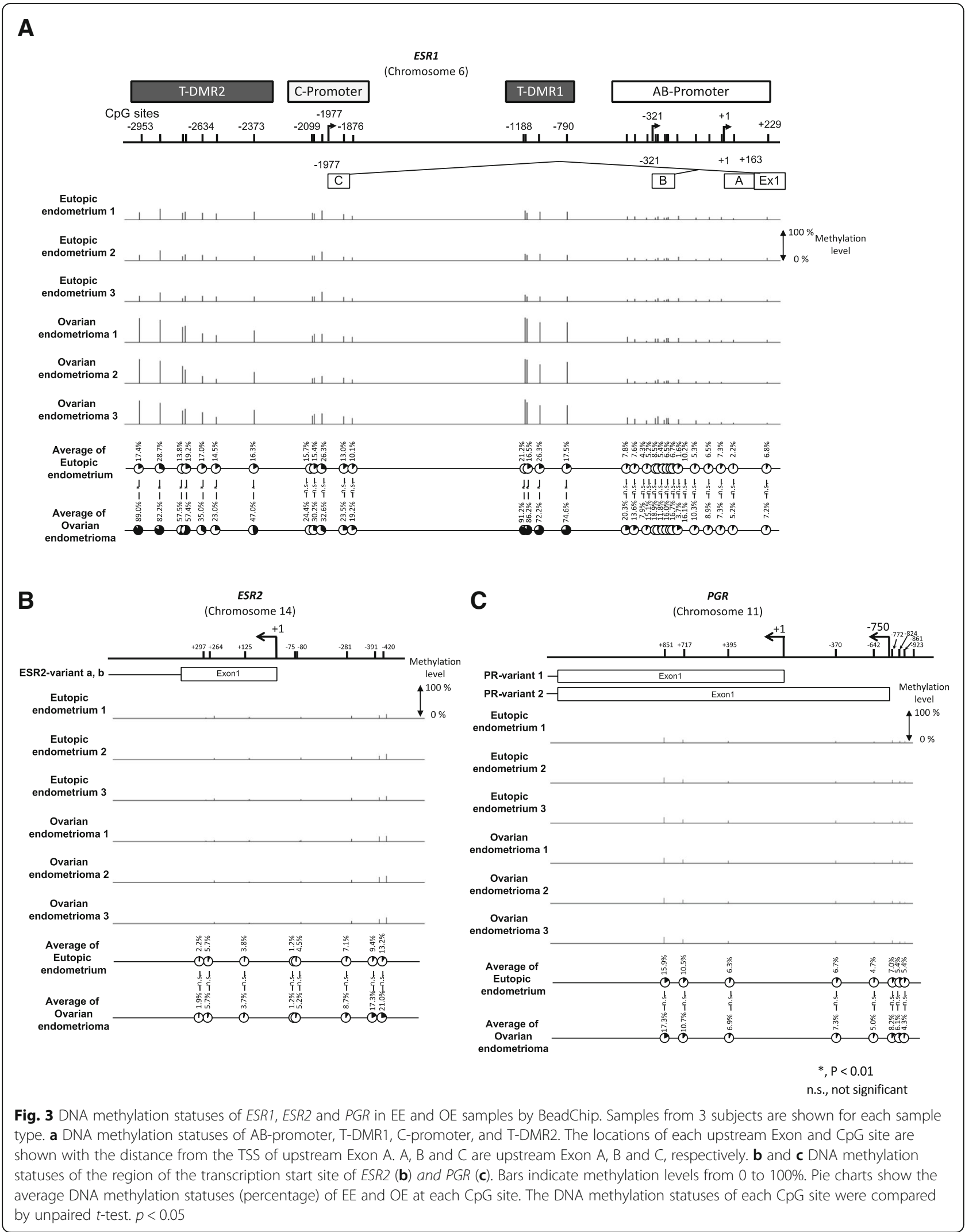




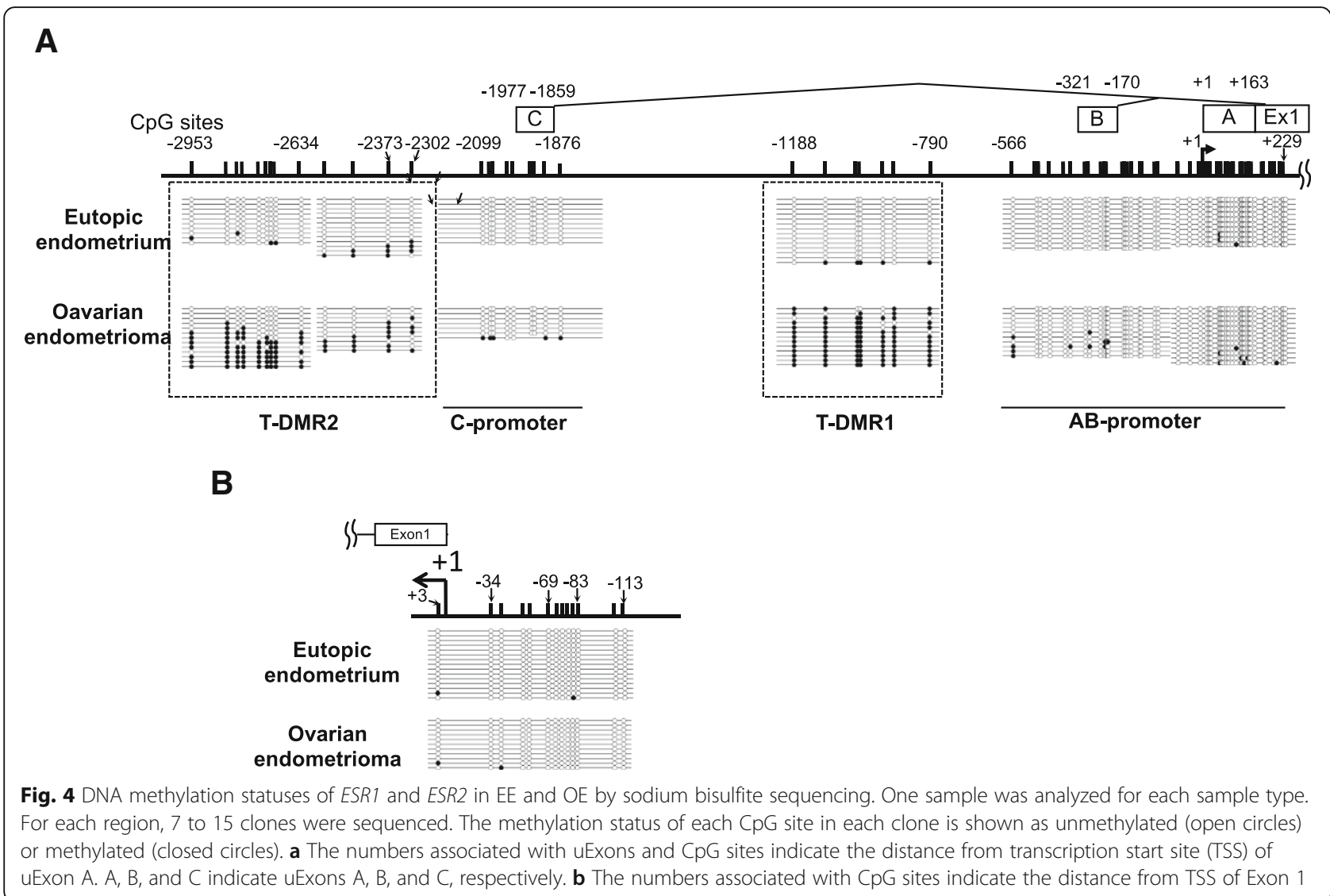

\section{Discussion}

We previously reported that DNA methylation of the T-DMRs of ESR1 regulates the expression of ESR1 in normal tissues [20]. The expression of ESR1 in the tissues with high DNA methylation at the T-DMRs was strongly suppressed compared with the expression of ESR1 in the tissues with low DNA methylation at the T-DMRs [20]. In the present study, ESR1 expression was lower and DNA methylation at the T-DMRs of ESR1 was higher in $\mathrm{OE}$ than in EE, indicating that the aberrant DNA methylation of the T-DMRs was associated with the impaired expression of ESR1 in OE. On the other hand, DNA methylation was not involved in the altered expressions of ESR2 and PGR in OE.

ESR1 is downregulated in cancer tissues such as breast cancers, and the downregulation was shown to be associated with DNA methylation of the promoter region of ESR1 [28-30]. However, Meyer et al. [7] found no significant difference in the DNA methylation status of ESR1 at the promoter region between EE and OE. We previously showed that methylation of the promoter region (not the T-DMR) of ESR1 was low in normal tissues with both high and low ESR1 expression [20,31]. These results indicated that the DNA methylation of the T-DMRs regulates the expression of ESR1 and there is no difference in DNA methylation of the promoter regions between tissues with different expression levels of ESR1 [20]. The present results show that the methylation levels of the promoter regions were kept low in both $\mathrm{OE}$ and $\mathrm{EE}$, which indicates that the impaired expression of ESR1 in OE is due to the T-DMRs and not the promoter region.

$E S R 2$, which is upregulated in $\mathrm{OE}$, has been shown to suppress ESR1 expression in endometriosis by binding to the ESR1 promoter $[8,32]$. Knockdown of ESR2 in endometriotic stromal cells increased the expression of ESR1 twofold, and overexpression of ESR2 in endometrial stromal cells reduced the expression of ESR1 by one-half $[8,32]$. In view of the huge difference in the expression levels of ESR1 between EE and OE (Fig. 2a), aberrant DNA methylation of the T-DMRs is likely a factor in the impaired expression of ESR1 in OE.

Xue et al. reported that the DNA methylation levels of the promoter region of ESR2 is high in endometrial stroma, while it is low in endometriotic stroma [22]. They concluded that the increased expression of ESR2 in endometriosis is caused by DNA hypomethylation of the promoter region (from - 163 to - 48) of ESR2 [22, 32]. However, $25 \%$ of their endometrial stroma samples also showed DNA hypomethylation. Because of this 
Table 1 Primer sets used in quantitative RT-PCR

\begin{tabular}{ll}
\hline Name & Forward \\
\hline ESR1 & \\
all variants & F: 5'-TGTGCAATG \\
variant1 (transcribed from uExon-A) & ACTATGCTCA-3' \\
& F: 5'-CTCGGGCT \\
variant2 (transcribed from uExon-B) & GTGCTCTTIT-3' \\
& F: 5'-GCCGTGAA \\
variant3 (transcribed from uExon-C) & ACTCAGCCTCTA-3' \\
& F: 5'-TGGAACATT \\
ESR2 & TCTGGAAAGACG-3' \\
variant-a/variant-b & \\
PGR & F: 5' -CTCGCTTC \\
variant1/variant2 & CTCAACAGGTG- 3' \\
variant2 & \\
& F: 5' -ACCAGCTCT \\
GAPDH & TGGTGCCTGT- 3' \\
& F: 5' -TCCCTCTGC \\
\hline
\end{tabular}

inconsistency, they may have shown only a difference among individuals. The present results show that DNA methylation in the region from -163 to -48 was low in both EE and OE. We also did not see any difference in DNA methylation in the regions upstream and downstream of this region; in these regions, both EE and OE were DNA hypomethylated. Our results suggest that DNA methylation is not involved in the upregulation of ESR2 in OE.

The expressions of $P G R$ variants 1 and 2 were significantly different between EE and OE. The present study showed no significant difference in the DNA methylation status between EE and $\mathrm{OE}$, indicating that DNA methylation is not associated with the differential expression of PGR. However, in breast cancer tissues, DNA methylation around the transcription start sites appears to have a role in the regulation of PGR expression $[25,26]$. Aberrant DNA methylation often occurs in the promoter region in a variety of cancers [33, 34]. In fact, we previously showed that DNA hypermethylation of the ESR1 promoter occurred only in cancer tissues and that this region remained hypomethylated in non-cancer tissues regardless of the ESR1 expression level [20]. Therefore, the difference between the previous reports and the present study may be due to a difference in the examined samples. Other mechanisms such as the binding of transcription factors may be associated with the differential expression of PGR between EE and OE.

\section{Conclusions}

We found that OE had aberrant DNA methylation in the T-DMRs of ESR1 and that the DNA methylation is associated with the impaired expression of ESR1 in OE.
On the other hand, it is unlikely that DNA methylation is associated with the altered expressions of ESR2 and $P G R$ in $\mathrm{OE}$.

\section{Methods}

\section{Tissue samples}

Specimens of EE and OE were obtained from 16 Japanese women. EE was obtained from patients who underwent surgery for uterine leiomyoma. The ages of the patients were $32.13+/-4.19$ years old (mean +/- SD; 26-40 years). OE tissue was obtained from patients who underwent cystectomy of $\mathrm{OE}$ during the proliferative phase $[10,16]$. The ages of the patients were $31.13+/-$ 5.08 years old (mean $+/-$ SD; 24-39 years old) and did not significantly differ from the patients with uterine leiomyoma $(p=0.67)$. None of the patients had received previous hormonal therapy. Specimens were dissected immediately after removal, immersed in liquid nitrogen and stored at $-80{ }^{\circ} \mathrm{C}$ until DNA/RNA extraction as previously reported $[35,36]$.

\section{Quantitative real-time RT-PCR (qRT-PCR)}

Total RNA was isolated from tissues using Isogen (Wako Pure Chemical Industries Ltd., Osaka, Japan). One $1 \mu \mathrm{g}$ total RNA was reverse-transcribed using a Quantitect Reverse Transcription Kit (Qiagen, Valencia, CA, USA) according to the manufacturer's protocol as previously reported [37]. To distinguish the transcribed variants derived from each upstream Exon (Fig. 1a), we synthesized three primer pairs as shown in Fig. 1a and Table 1. ESR2 is also known to have two major variants, variant $\mathrm{a}$ and $\mathrm{b}$ (Fig. 1b). We made a primer pair that amplifies both variants (Fig. $1 \mathrm{~b}$ and Table 1). PGR has two isoforms, $P R-A$ and $P R-B$, encoded by $P G R$-variants 1 and 2 , respectively (Fig. $1 \mathrm{c}$ ). Since all nucleotides in $P G R$-variant 1 are included in $P G R$-variant 2, the primer pair designed for $P G R$ variant 1 also amplified $P G R$ variant 2 . We designed two primer pairs. One detected the combined expression level of PGR variants 1 and 2, and the other detected PGR-variant 2 specifically (Fig. 1c and Table 1). A primer pair for glyceraldehyde-3-phosphate dehydrogenase $(G A P D H)$ was used as an internal control (Table 1). Real-time qRT-PCR was performed with 5 specimens each of EE and OE using SYBR Premix Ex Taq (Takara, Ohtsu, Japan) and a LightCycler (Roche Applied Science, Basel, Switzerland). All samples were run in duplicate. The relative quantity of cDNA was calculated with the $\Delta \Delta \mathrm{Ct}$ method. Melting curves of the products were obtained after cycling by a stepwise increase of temperature from 55 to $95^{\circ} \mathrm{C}$.

Illumina Infinium HumanMethylation450k BeadChip assay Genomic DNA was isolated from $20 \mathrm{mg}$ of frozen tissue using a Qiagen Genomic DNA kit (Qiagen). DNA 
Table 2 Primer sets used in sodium bisulfite sequencing analysis

\begin{tabular}{|c|c|c|}
\hline Name & Forward & Reverse \\
\hline ESR1_Region I (- 129 to + 279) & F: 5'-GTTGTGTTTGGAGTGATGTTTAAGTT-3' & R: 5'-CAATAAAACCATCCCAAATACTTTA-3' \\
\hline ESR1_Region II (- 670 to -94$)$ & F: 5'-GGAAGGGTTTATTATTTTGGGAGTA-3' & R: 5'-TAACATTAACTTAAACATCACTCC-3' \\
\hline ESR1_Region III (- 1298 to - 731) & F: 5'-TTGGGTGTTTGGGATAGTAATTAAA-3' & R: 5'-CTTAATCCCATTAAAAATTCTCAT-3' \\
\hline ESR1_Region IV (- 2227 to - 1756) & F: 5'-TAGTTITAAGGGTAGGGGTAAAGG-3' & R: 5'-CAACAATCCTCATCTCCCTACTAAA-3' \\
\hline ESR1_Region V (- 2595 to - 2241) & F: 5'-TATTTATGGAAAGGTTTGTGGGTTT-3' & R: 5'-TACTTTCTACTACCACCCCAAACAA-3' \\
\hline ESR1_Region VI (- 2981 to - 2573) & F: 5'-AATTGTATAGTGTTTAAGGGTTAGAGA-3' & R: 5'-ACCCACAAACCTITCCATAAATAAC-3' \\
\hline ESR2 $(-113$ to +3$)$ & F: 5'-ATTATTTTGTGGGTGGATTAGGAG-3' & R: 5'-AACCCCTTCTTCCTTTTAAAAACC-3' \\
\hline
\end{tabular}

methylation was analyzed with an Illumina Infinium assay with the HumanMethylation450 BeadChip (Illumina, San Diego, CA, USA), which interrogates a total of $482,421 \mathrm{CpG}$ sites from the distal promoter regions of the transcription start sites to the 3'-UTR of consensus coding sequences. Methylated and unmethylated signals were used to compute beta values, which are quantitative scores of the DNA methylation levels, ranging from 0 (completely unmethylated) to 1 (completely methylated). The BeadChip was scanned on a BeadArray Reader (Illumina) according to the manufacturer's instructions. CpG sites with "detection p values" > 0.05 (computed from the background based on negative controls) and CpG sites on the Y chromosome were eliminated from further analysis, leaving 482,005 CpGs valid for use with the nine samples tested.

\section{Sodium bisulfite sequencing}

Bisulfite reactions were performed using an EpiTect $\mathrm{Bi}$ sulfite Kit (Qiagen) with the following temperature conditions: $95^{\circ} \mathrm{C}$ for $5 \mathrm{~min}, 65^{\circ} \mathrm{C}$ for $85 \mathrm{~min}, 95^{\circ} \mathrm{C}$ for $5 \mathrm{~min}$ and $65^{\circ} \mathrm{C}$ for $175 \mathrm{~min}$ as previously reported [38]. The bisulfite-converted DNA was amplified by PCR using the primer pairs for ESR1 and ESR2 shown in Table 2 using the following thermocycling conditions: $95^{\circ} \mathrm{C}$ for $10 \mathrm{~min}$, and $40 \mathrm{cycles}$ of $94{ }^{\circ} \mathrm{C}$ for $30 \mathrm{~s}, 60^{\circ} \mathrm{C}$ for $30 \mathrm{~s}$ and $72^{\circ} \mathrm{C}$ for $1 \mathrm{~min}$ followed by $10 \mathrm{~min}$ of final extension at $72^{\circ} \mathrm{C}$. The resulting products were cloned into a pGEM-T easy vector (Promega, Tokyo, Japan). The vectors were sequenced using a BigDye Terminator V3.1 Kit (Applied Biosystems, Foster City, CA, USA) and a 3130xl Genetic Analyzer (Applied Biosystems) as previously reported [20]. QUMA (http://quma.cdb.riken.jp/) was used to analyze the bisulfite sequencing data [39].

\section{Statistical analyses}

DNA methylation and mRNA Expression levels of the two groups were compared with unpaired $t$-tests using SPSS for Windows version 11 (SPSS Inc., Chicago, IL, USA).

\section{Abbreviations}

EE: Eutopic endometrium; ERa: Estrogen receptor alpha; ERß: Estrogen receptor beta; ESR1: Estrogen receptor 1; ESR2: Estrogen receptor 2;
GAPDH: Glyceraldehyde-3-phosphate dehydrogenase; OE: Ovarian endometrioma; PGR: Progesterone receptor; qRT-PCR: Quantitative real-time RT-PCR; T-DMR: Tissue-dependent and differentially methylated region

\section{Acknowledgements}

Not applicable.

\section{Funding}

This work was supported in part by JSPS KAKENHI Grants C16K11142 C16K11091, C18K09262, C17K11239, C18K09230, 18 K16801 and 18 K16772 for Scientific Research from the Ministry of Education, Science, and Culture, Japan.

Availability of data and materials

The datasets used and analyzed during the current study are available from the corresponding author on reasonable request

\section{Authors' contributions}

RM is the first/corresponding author, and participated in the study designing, supervision, experiment, analysis of BeadChip, statistical analysis and interpretation of the data and drafting of the manuscript. YM, SS, MO, IT, MS, YS, HT and TT participated in the measurement of the levels of mRNA expression and DNA methylation, interpretation of the measured data, and drafting the manuscript. IT and MS participated in the analysis of BeadChip and statistical analysis. HT and NS conceived, designed and supervised the study and participated in drafting the manuscript. All authors read and approved the final manuscript.

\section{Ethics approval and consent to participate}

This study was reviewed and approved by the Institutional Review Board of Yamaguchi University Graduate School of Medicine. Written informed consent was obtained from the participants before the collection of any samples, and the specimens were irreversibly de-identified. All experiments involving the handling of human tissues were performed in accordance with Tenets of the Declaration of Helsinki.

\section{Consent for publication}

Written informed consent for publication was obtained from the participants before the collection of any samples.

\section{Competing interests}

The authors declare that they have no competing interests.

\section{Publisher's Note}

Springer Nature remains neutral with regard to jurisdictional claims in published maps and institutional affiliations.

Received: 18 November 2018 Accepted: 28 January 2019

Published online: 06 February 2019

\section{References}

1. Rogers PA, D'Hooghe TM, Fazleabas A, Gargett CE, Giudice LC, Montgomery GW, et al. Priorities for endometriosis research: recommendations from an international consensus workshop. Reprod Sci. 2009;16(4):335-46. 
2. Nakamura M, Yamashita Y, Hayashi A, Saito N, Yu M, Hayashi M, et al. Analyzing the risk factors for a diminished oocyte retrieval rate under controlled ovarian stimulation. Reprod Med Biol. 2017;16(1):40-4.

3. Jia M, Dahlman-Wright K, Gustafsson JA. Estrogen receptor alpha and beta in health and disease. Best Pract Res Clin Endocrinol Metab. 2015; 29(4):557-68

4. Paterni I, Granchi C, Katzenellenbogen JA, Minutolo F. Estrogen receptors alpha (ERalpha) and beta (ERbeta): subtype-selective ligands and clinical potential. Steroids. 2014;90:13-29.

5. Hewitt SC, Harrell JC, Korach KS. Lessons in estrogen biology from knockout and transgenic animals. Annu Rev Physiol. 2005;67:285-308.

6. Fujimoto J, Hirose R, Sakaguchi H, Tamaya T. Expression of oestrogen receptor-alpha and -beta in ovarian endometriomata. Mol Hum Reprod. 1999:5(8):742-7

7. Meyer JL, Zimbardi D, Podgaec S, Amorim RL, Abrao MS. Rainho CA. DNA methylation patterns of steroid receptor genes ESR1, ESR2 and PGR in deep endometriosis compromising the rectum. Int J Mol Med. 2014;33(4):897904.

8. Trukhacheva $E$, Lin Z, Reierstad S, Cheng YH, Milad M, Bulun SE. Estrogen receptor (ER) beta regulates ERalpha expression in stromal cells derived from ovarian endometriosis. J Clin Endocrinol Metab. 2009:94(2):615-22.

9. Kim M, Costello J. DNA methylation: an epigenetic mark of cellular memory. Exp Mol Med. 2017;49(4):e322.

10. Yamagata Y, Nishino K, Takaki E, Sato S, Maekawa R, Nakai A, et al. Genomewide DNA methylation profiling in cultured eutopic and ectopic endometrial stromal cells. PLoS One. 2014;9(1):e83612.

11. Hsiao KY, Wu MH, Tsai SJ. Epigenetic regulation of the pathological process in endometriosis. Reprod Med Biol. 2017;16(4):314-9.

12. Koike N, Higashiura Y, Akasaka J, Uekuri C, Ito F, Kobayashi H. Epigenetic dysregulation of endometriosis susceptibility genes (review). Mol Med Rep. 2015;12(2):1611-6.

13. Sultana S, Kajihara T, Mizuno Y, Sato T, Oguro T, Kimura M, et al. Overexpression of microRNA-542-3p attenuates the differentiating capacity of endometriotic stromal cells. Reprod Med Biol. 2017;16(2):170-8.

14. Burney RO, Giudice LC. Pathogenesis and pathophysiology of endometriosis. Fertil Steril. 2012;98(3):511-9.

15. Sokalska A, Anderson M, Villanueva J, Ortega I, Bruner-Tran KL, Osteen KG, et al. Effects of simvastatin on retinoic acid system in primary human endometrial stromal cells and in a chimeric model of human endometriosis. J Clin Endocrinol Metab. 2013:98(3):E463-71.

16. Yamagata Y, Takaki E, Shinagawa M, Okada M, Jozaki K, Lee L, et al. Retinoic acid has the potential to suppress endometriosis development. J Ovarian Res. 2015;8:49

17. Shiota K, Kogo Y, Ohgane J, Imamura T, Urano A, Nishino K, et al. Epigenetic marks by DNA methylation specific to stem, germ and somatic cells in mice. Genes Cells. 2002;7(9):961-9.

18. Sakamoto H, Suzuki M, Abe T, Hosoyama T, Himeno E, Tanaka S, et al. Cell typespecific methylation profiles occurring disproportionately in $\mathrm{CpG}$-less regions that delineate developmental similarity. Genes Cells. 2007;12(10):1123-32.

19. Yagi S, Hirabayashi K, Sato S, Li W, Takahashi Y, Hirakawa T, et al. DNA methylation profile of tissue-dependent and differentially methylated regions (T-DMRs) in mouse promoter regions demonstrating tissue-specific gene expression. Genome Res. 2008;18(12):1969-78.

20. Maekawa R, Sato S, Okada M, Lee L, Tamura I, Jozaki K, et al. Tissue-specific expression of estrogen receptor 1 is regulated by DNA methylation in a TDMR. Mol Endocrinol. 2016;30(3):335-47.

21. Sato S, Maeda C, Hattori N, Yagi S, Tanaka S, Shiota K. DNA methylationdependent modulator of Gsg2/Haspin gene expression. J Reprod Dev. 2011; 57(4):526-33.

22. Xue Q, Lin Z, Cheng YH, Huang CC, Marsh E, Yin P, et al. Promoter methylation regulates estrogen receptor 2 in human endometrium and endometriosis. Biol Reprod. 2007;77(4):681-7.

23. Bedaiwy MA, Dahoud W, Skomorovska-Prokvolit Y, Yi L, Liu JH, Falcone T, et al. Abundance and localization of progesterone receptor isoforms in endometrium in women with and without endometriosis and in peritoneal and ovarian endometriotic implants. Reprod Sci. 2015;22(9):1153-61.

24. Attia GR, Zeitoun K, Edwards D, Johns A, Carr BR, Bulun SE. Progesterone receptor isoform $A$ but not $B$ is expressed in endometriosis. J Clin Endocrinol Metab. 2000;85(8):2897-902.

25. Pathiraja TN, Shetty PB, Jelinek J, He R, Hartmaier R, Margossian AL, et al. Progesterone receptor isoform-specific promoter methylation: association of
PRA promoter methylation with worse outcome in breast cancer patients. Clin Cancer Res. 2011:17(12):4177-86.

26. Mc Cormack O, Chung WY, Fitzpatrick P, Cooke F, Flynn B, Harrison M, et al. Progesterone receptor $B$ (PRB) promoter hypermethylation in sporadic breast cancer: progesterone receptor B hypermethylation in breast cancer. Breast Cancer Res Treat. 2008;111(1):45-53.

27. Reid G, Denger S, Kos M, Gannon F. Human estrogen receptor-alpha: regulation by synthesis, modification and degradation. Cell Mol Life Sci. 2002;59(5):821-31.

28. Lapidus RG, Ferguson AT, Ottaviano YL, Parl FF, Smith HS, Weitzman SA, et al. Methylation of estrogen and progesterone receptor gene $5^{\prime} \mathrm{CpG}$ islands correlates with lack of estrogen and progesterone receptor gene expression in breast tumors. Clin Cancer Res. 1996;2(5):805-10.

29. Yan L, Yang $X$, Davidson NE. Role of DNA methylation and histone acetylation in steroid receptor expression in breast cancer. J Mammary Gland Biol Neoplasia. 2001;6(2):183-92.

30. Yoshida T, Eguchi H, Nakachi K, Tanimoto K, Higashi Y, Suemasu K, et al. Distinct mechanisms of loss of estrogen receptor alpha gene expression in human breast cancer: methylation of the gene and alteration of transacting factors. Carcinogenesis. 2000;21(12):2193-201.

31. Asada H, Yamagata Y, Taketani T, Matsuoka A, Tamura H, Hattori N, et al. Potential link between estrogen receptor-alpha gene hypomethylation and uterine fibroid formation. Mol Hum Reprod. 2008;14(9):539-45.

32. Bulun SE, Cheng YH, Pavone ME, Xue Q, Attar E, Trukhacheva E, et al. Estrogen receptor-beta, estrogen receptor-alpha, and progesterone resistance in endometriosis. Semin Reprod Med. 2010;28(1):36-43.

33. Hattori N, Ushijima T. Compendium of aberrant DNA methylation and histone modifications in cancer. Biochem Biophys Res Commun. 2014;455:3.

34. Barrow TM, Michels KB. Epigenetic epidemiology of cancer. Biochem Biophys Res Commun. 2014;455:70.

35. Maekawa R, Yagi S, Ohgane J, Yamagata Y, Asada H, Tamura I, et al. Diseasedependent differently methylated regions (D-DMRs) of DNA are enriched on the $X$ chromosome in uterine leiomyoma. J Reprod Dev. 2011:57(5):604-12.

36. Maekawa R, Taketani T, Mihara Y, Sato S, Okada M, Tamura I, et al. Thin endometrium transcriptome analysis reveals a potential mechanism of implantation failure. Reprod Med Biol. 2017;16(2):206-27.

37. Maekawa R, Sato S, Yamagata Y, Asada H, Tamura I, Lee L, et al. Genomewide DNA methylation analysis reveals a potential mechanism for the pathogenesis and development of uterine leiomyomas. PLoS One. 2013;8(6): e66632.

38. Sato S, Maekawa R, Yamagata Y, Asada H, Tamura I, Lee L, et al. Potential mechanisms of aberrant DNA hypomethylation on the $x$ chromosome in uterine leiomyomas. J Reprod Dev. 2014;60(1):47-54.

39. Kumaki Y, Oda M, Okano M. QUMA: quantification tool for methylation analysis. Nucleic Acids Res. 2008;36(Web Server issue):W170-5.

Ready to submit your research? Choose BMC and benefit from:

- fast, convenient online submission

- thorough peer review by experienced researchers in your field

- rapid publication on acceptance

- support for research data, including large and complex data types

- gold Open Access which fosters wider collaboration and increased citations

- maximum visibility for your research: over $100 \mathrm{M}$ website views per year

At BMC, research is always in progress.

Learn more biomedcentral.com/submission 